\title{
Precise measurement of effective oxygen diffusivity for microporous media containing moisture by review of galvanic cell oxygen absorber configuration
}

\author{
Ryo Koresawa $^{1}$ and Yoshio Utaka ${ }^{2}$ \\ ${ }^{1}$ Graduate School of Engineering, Yokohama National University \\ 79-5 Tokiwadai, Hodogaya, Yokohama, Kanagawa, Japan \\ ${ }^{2}$ Faculty of Engineering, Yokohama National University \\ 79-5 Tokiwadai, Hodogaya, Yokohama, Kanagawa, Japan
}

The performance of polymer electrolyte fuel cells is influenced by moisture control in their gas diffusion layer (GDL). Therefore, to achieve suitable control, it is necessary to clarify the mass transfer characteristics within a GDL by high precision measurement of oxygen diffusivity. We have previously proposed that measurement of the effective oxygen diffusivity in a GDL containing moisture can be achieved using a galvanic cell oxygen absorber and demonstrated this to be an effective technique for the measurement of microporous media. However, the diffusion resistance of a single dry GDL is low, so that the margin of error in the oxygen diffusivity measurement is high. In this study, high precision measurement of the oxygen diffusivity in a GDL was developed further by analysis of the major error factors and modification of the measurement apparatus configuration. The results indicate a reduction in the maximum measurement error from 50 to $20 \%$ for a dry GDL with minimal diffusion resistance.

Key Words: effective oxygen diffusivity, galvanic cell oxygen absorber, gas diffusion layer, polymer electrolyte fuel cell 


\begin{tabular}{|ll|}
\hline Nomenclature \\
$A$ & area of carbon electrode film $\left[\mathrm{m}^{2}\right]$ \\
$C$ & mass transfer conductance $\left[\mathrm{m}^{3} / \mathrm{s}\right]$ \\
$D$ & oxygen diffusivity $\left[\mathrm{m}^{2} / \mathrm{s}\right]$ \\
$E$ & output voltage $[\mathrm{mV}]$ \\
$F$ & Faraday constant $[\mathrm{s} \cdot \mathrm{A} / \mathrm{mol}]$ \\
$I$ & output current $[\mathrm{A}]$ \\
$j$ & mass flux of oxygen $\left[\mathrm{kg} /\left(\mathrm{m}^{2} \mathrm{~s}\right)\right]$ \\
$p$ & pressure $[$ atm $]$ \\
$R$ & mass transfer resistance $\left[\mathrm{s} / \mathrm{m}^{3}\right]$ \\
$S$ & average saturation in $\mathrm{GDL}[\%]$ \\
Greek symbols & \\
$\rho$ & density $\left[\mathrm{kg} / \mathrm{m}^{3}\right]$ \\
$\omega$ & mass fraction of oxygen $[-]$ \\
Subscripts & \\
ave & average \\
C & carbon electrode film \\
eff & effective \\
exp & experimental \\
Gas & gas \\
GDL & gas diffusion layer \\
Glv & galvanic cell \\
num & numerical \\
$\mathrm{O}_{2}$ & oxygen \\
1 & effect of liquid surface difference \\
2 & effect of distance between films \\
\hline
\end{tabular}




\section{Introduction and summary of relevant former studies}

The objective of this study is to improve the precision of effective oxygen diffusivity measurements for microporous media containing moisture, such as the gas diffusion layer (GDL) of a polymer electrolyte fuel cell (PEFC), using a galvanic cell oxygen absorber.

PEFCs are expected to be used as lightweight and compact power sources for vehicles, due to their high power densities and superior start-up characteristics at low operating temperatures. PEFCs have also become commercially available as domestic cogeneration systems. However, a reduction of the system cost and higher output power density are still required. Degradation of performance due to the amount of moisture is an especially important problem. For example, water or condensation from humidified vapor accumulates in the gas diffusion layer (GDL) and gas channels of the separator, which hinders diffusion of the reactant gas under conditions of high humidity and high current density. In contrast, proton conductivity in the polymer electrolyte film decreases when the cell becomes dry under low humidity conditions. Therefore; it is important to control the liquid water in PEFCs and to clarify the oxygen diffusion characteristics in a GDL containing moisture at the cathode side, where the influence of generated water is most significant. Thus, it is necessary to measure the effective oxygen diffusion coefficient for the GDL with a high degree of accuracy.

Various methods for measuring the effective diffusion coefficient in porous media have been previously reported. For example, the diaphragm method was used by Henry et al. [1] and Masamune and Smith [2]. This method is used to determine the effective diffusivity by directly measuring the gas permeability through a porous medium. Diluted gases with different concentrations are passed through the channels that sandwich the porous medium, and the difference in concentration between the upper and lower flows is measured. However, this method is complex and requires a considerable amount of time to obtain highly accurate measurements. A reaction-diffusion system was used by Wakao and Funaki [3], and a separation membrane method was employed by Hamai and Mitani [4] and Gibilaro and Waldram [5]. The reaction and adsorption methods were used to measure the rate of reaction or rate of adsorption of a gas on the surface of a porous medium. In addition, mass transport in a porous material was modeled on the basis of certain assumptions to determine the relationship between the mass transfer rate and the effective diffusivity. The effective diffusivity is quantified from the change in the measured carrier gas concentration with elapsed time. Accordingly, the accuracy of the diffusion coefficient is strongly dependent not only on the measurement accuracy, but also on the simulation model employed. Further, an oxygen sensor with an yttria-stabilized zirconia (YSZ) electrolyte was employed by Mezedur et al. [6] to measure gas diffusivity in porous electrode catalysts based on oxygen ion transfer in the YSZ electrolyte. The effective oxygen diffusivity was determined by simultaneously measuring the permeating oxygen flux in the porous medium and the oxygen concentration at its surface. However, this sensor is generally used at temperatures above $300{ }^{\circ} \mathrm{C}$, and it is difficult to simply and precisely measure gas diffusivity under wet conditions where liquid water is present in the micropores.

Recently, the effective diffusivity of the GDL in PEFCs was experimentally measured. Kramer et al. [7] and Flückiger et al. [8] obtained the effective diffusivity using electrochemical diffusimetry. Electrochemical impedance spectroscopy was applied to measure the effective ionic conductivity of an electrolyte-soaked GDL, and the effective diffusivity, which was expressed as the ratio of the bulk diffusion coefficient to the diffusion coefficient for the GDL, was determined by taking advantage of the analogy between Fick's law and Ohm's law. The effective diffusivity in a dry GDL was measured by Baker et al. [9] and that in a wet GDL by Hwang and Weber [10] using the limiting current density technique. Hwang and Weber [10] measured the effective diffusivity of hydrogen through argon in a binary mixture with a sample containing moisture. Zamel et al. [11] and Chan et al. [12] measured the effective diffusivity in a dry GDL using a modified Loschmidt cell and applying the one-dimensional Fick's law. However, there have been few studies that have measured the effective diffusivity in a sample containing moisture.

Through research on water management in PEFCs, the authors have developed a method to measure the oxygen diffusivity of microporous media containing moisture [13,14]. Utaka et al. [13] evaluated the gas diffusion characteristics of a dry GDL using a method that employed a galvanic cell oxygen absorber, where multi-layered GDLs with a larger diffusion resistance were used to obtain the precise oxygen diffusivity. The galvanic cell oxygen absorber was uniquely designed by 
upgrading a galvanic cell oxygen sensor that is practically used to detect the oxygen concentration and measure a comparatively large oxygen flux. However, diffusivity measurement of a single GDL containing moisture is required under conditions similar to those during practical operation. The galvanic cell oxygen absorber with peripheral equipment and the measurement method were further improved to evaluate the oxygen diffusion characteristics in a single GDL with/without the presence of moisture [14]. This method was demonstrated to be effective for measuring a single GDL containing moisture and the measurement error factors were also examined. However, further improvement of the diffusivity measurement precision is necessary, especially for investigating dry single GDLs that have the lowest diffusion resistance to moisture saturation. It is important to consider the effect of the concentration distributions in the vicinity of both GDL surfaces due to a reduction of mass transfer resistance in the GDL during a single GDL measurement. However, it is difficult to correctly measure the concentration distributions at both surfaces of the sample. Therefore, a special method was required for deriving the diffusion coefficient with sufficient accuracy. The authors have previously developed a comparison method [14], where the apparent mass transfer conductance of a standard test sample with a known diffusion coefficient is measured consecutively under the same experimental conditions with and without the sample. Using the standard sample measurement, the effective oxygen diffusion coefficient for the test sample was determined by adopting the bulk characteristics of oxygen reaction in the galvanic cell oxygen absorber as a variation of the effective diffusion resistance. Furthermore, this method was applied to measure the diffusivity in a specially devised GDL containing moisture [15]. In such an evaluation, error analysis indicated that stability of the output characteristics of the galvanic cell oxygen absorber was the most important issue for achieving high precision measurement of the oxygen diffusivity with the comparison method. Heretofore, the measurement precision with this method was guaranteed by using only results obtained when the change in the output of the galvanic cell oxygen absorber under the same conditions was low [14]. However, there was still a limitation on the measurement accuracy. The measurement results for the effective diffusivity in a dry GDL had a spread of $\pm 50 \%$ around the average value, while there a lower data spread for the diffusivity in a moist GDL with larger diffusion resistance.

In this study, those factors that should be improved for output stability of the galvanic cell oxygen absorber are examined and the diffusivity measurement accuracy is improved by upgrading the configuration of the galvanic cell oxygen absorber measurement apparatus.

\section{Galvanic cell oxygen absorber and effective oxygen diffusivity measurement}

\subsection{Fundamentals of galvanic cell oxygen absorber apparatus}

Figure 1 shows a schematic diagram of the experimental galvanic cell oxygen absorber apparatus used to measure the effective oxygen diffusivity in a GDL. The absorber consists of a carbon electrode film (cathode), a lead electrode plate (anode), and an electrolyte of which the principal ingredient is potassium hydroxide solution. An oxygen limiting filter with small holes is located on the side of the carbon electrode film exposed to the atmosphere in order to limit the oxygen permeation area and adjust the oxygen flux. The hydrophobic carbon film cathode acts as an oxygen penetration diaphragm that separates the atmosphere and electrolyte, but allows oxygen in the atmosphere to penetrate and undergo electrochemical reaction. The electrochemical reactions at the two electrodes are expressed as:

$$
\text { [Cathode] } \mathrm{O}_{2}+2 \mathrm{H}_{2} \mathrm{O}+4 \mathrm{e}^{-} \rightarrow 4 \mathrm{OH}^{-}
$$

$$
\text { [Anode] } 2 \mathrm{~Pb} \rightarrow 2 \mathrm{~Pb}^{2+}+4 \mathrm{e}^{-}
$$

In the cathode, the reduction of oxygen occurs when oxygen penetrates the cathode and dissolves into the electrolyte. As a result, the experimental apparatus effectively functions as an oxygen absorber. Corresponding to this process, the lead anode is oxidized according to the chemical reaction given in Eq. (2). Lead dissolves in the alkaline electrolyte and water is produced in the same quantity as the amount consumed at the cathode, according to:

$$
\mathrm{Pb}^{2+}+3 \mathrm{OH}^{-} \rightarrow \mathrm{HPbO}_{2}^{-}+\mathrm{H}_{2} \mathrm{O}
$$


The quantity of oxygen gas consumed by the electrochemical reaction at the cathode can be obtained from the output current $I[\mathrm{~A}]$ of the oxygen absorber using Eq. (4). Thus, the output current resulting from oxygen reduction due to reaction at the cathode surface is determined by measuring the voltage drop across an in-circuit resistor from the electromotive force of the oxygen absorber.

$$
j_{\mathrm{O}_{2}}=32 \times 10^{-3} \times \frac{I}{4 F} \times \frac{1}{A}
$$

where $j_{\mathrm{O}_{2}} \mathrm{~kg} /\left(\mathrm{m}^{2} \cdot \mathrm{s}\right)$ is the oxygen mass flux, $F(\mathrm{~s} \cdot \mathrm{A}) / \mathrm{mol}$ is the Faraday constant and $A\left(\mathrm{~m}^{2}\right)$ is the cross-sectional area of the carbon electrode film. The measurement apparatus was covered with a box, of which the top was a gas permeable film, to prevent flow disturbance, as shown in Fig. 2.

Details of the porous media test material are given in Table 1. Carbon paper (Toray, TGP-H-120) is typically used as a GDL in PEFCs. The evacuation impregnation method was used to fill the test material with water, which involves filling pure water into the voids of the sample by placing the sample in pure water and decompressing it in a vacuum vessel.

\subsection{Method for determining effective oxygen diffusivity}

The apparatus configuration along the direction of the oxygen path is two-dimensionally axisymmetric; therefore, the distribution and oxygen flux were calculated numerically to determine the effective oxygen diffusivity. The computational fluid dynamics software ANSYS Fluent 13.0 was used for numerical calculation. The following sections describe the method used for determination of the effective oxygen diffusivity.

\subsubsection{Basic equations and boundary conditions}

A cylindrical coordinate system was utilized, considering the axial symmetry of the measurement apparatus, and the two-dimensional concentration distributions of the test surfaces were calculated. For the calculation, it was assumed that the air outside the calculation domain was a mixture of oxygen and nitrogen with mass fractions of 0.2324 and 0.7676 , respectively. Calculations for continuity, momentum and mass conservation in the domain corresponding to the measurement system shown in Fig. 3 were performed. No mass flux was assumed and non-slip conditions were applied at the hatched area. An atmospheric concentration of air and free exit flow conditions were set for the area bounded by broken lines. There are four main calculation domains: the galvanic cell for oxygen absorption with the carbon electrode film (cathode), the oxygen limiting filter, the electrolyte and lead electrode plate (anode) ("oxygen reaction area" is used as an abbreviation) at $z \leq 0$, the oxygen permeation area at $0<z \leq 2.9$, the test material installation area at $2.9<z \leq 3.9$, and the area open to the atmosphere at $3.9<z \leq 29.4$. The air-side surface of the carbon electrode film is at $z=0$. The porous medium is attached to the test material installation area. There was little difference between the calculated results for effective oxygen diffusivity of the porous medium considering diffusion without convection and those under the conditions given; therefore, the former calculation method was adopted for simplicity. The basic equation and boundary conditions are given in the following.

The oxygen diffusion in a binary mixture of oxygen and nitrogen follows Fick's law of diffusion and is shown in Eq. (5). Details regarding determination of the boundary conditions in the oxygen reaction area denoted by Eq. (10) are described later.

$$
j_{\mathrm{O}_{2}}=-\rho D_{\mathrm{O}_{2}} \nabla \omega_{\mathrm{O}_{2}}
$$

Here, $j_{\mathrm{O}_{2}}$ is the oxygen mass flux, $\rho$ is the density, $D_{\mathrm{O}_{2}}$ is the oxygen diffusivity and $\omega_{\mathrm{O}_{2}}$ is the mass fraction of oxygen. The boundary conditions are shown in Eqs. (6) to (10):

$$
r=16, \quad 17.4 \leq z \leq 29.4 ; \omega_{\mathrm{O}_{2}}=0.2324
$$




$$
\begin{aligned}
& r \leq 16, \quad z=29.4 ; \omega_{\mathrm{O}_{2}}=0.2324 \\
& (9 \leq r \leq 16, z=17.4),(2.5 \leq r \leq 9, z=3.9),(2.5 \leq r \leq 11, z=2.9) ; \partial \omega_{\mathrm{O}_{2}} / \partial z=0 \\
& (r=9,3.9 \leq z \leq 17.4),(r=11,0 \leq z \leq 2.9),(r=2.5,2.9 \leq z \leq 3.9) ; \partial \omega_{\mathrm{O}_{2}} / \partial r=0
\end{aligned}
$$

For two-dimensional calculation, boundary condition of electrochemical reaction is approximated by apparent mass transfer conductance $C_{\mathrm{c}}$ at $\mathrm{z}=0$.

$$
(r \leq 11, z=0) \quad ; \quad j_{\mathrm{C}}=C_{\mathrm{C}} \Delta \omega_{\mathrm{O}_{2}}, C_{\mathrm{C}}=f\left(j_{\exp }\right), \omega_{\mathrm{O}_{2}}=0
$$

For three-dimensional calculation, it is important to consider the distribution of oxygen concentration and flux. The apparent diffusion coefficient $D_{\mathrm{c}}$ was adopted in the region of electrode and anode lead metal $(-0.3<z \leq 0)$ as shown in Eq.(11) and (12).

$$
\begin{aligned}
& (r \leq 11,-0.3<z \leq 0): \quad j_{\mathrm{C}}=-\rho D_{\mathrm{C}} \nabla \omega_{\mathrm{O}_{2}} \\
& r \leq 11, z=-0.3: \omega_{\mathrm{O}_{2}}=0
\end{aligned}
$$

\subsubsection{Determination of boundary conditions for bulk characteristics of oxygen reaction and derivation of oxygen diffusivity in GDL containing moisture}

The phenomena occurring in the galvanic cell oxygen absorber were determined from the mass diffusion and electrochemical reactions with electricity generation. For the purposes of this study, it was not efficient to strictly consider the complex effects of chemical reactions in the numerical calculation; therefore, a boundary condition for obtaining a suitable approximation of the oxygen electrochemical reaction and diffusion in the electrolyte of the oxygen reaction area was adopted as a mass transfer conductance determined by the measurement of standard samples. An apparent oxygen mass conductance was employed to approximate the effects of chemical reactions in the oxygen absorber; the mass fraction of oxygen was set to zero at the surface of the carbon electrode film and it was assumed that mass transfer occurred in the oxygen reaction area $z \leq 0$, as shown in Fig. 3. The apparent conductance of the oxygen reaction area was expressed as a function of the oxygen mass flux.

The comparison method [14] was used to determine the oxygen diffusivity in the porous medium as described in Section 1; therefore, a comparison of the experimentally measured results with the numerical results for the same system with a material of known oxygen diffusivity (standard sample) was required to set the boundary conditions for the oxygen reaction area. Air in a tube with a given length was used as a standard sample of which the diffusion conductance could be calculated exactly. Six tubes with different lengths (15.0-100.0 mm) and mass transfer resistances that cover those of the test samples were prepared to vary the oxygen diffusion resistance. The relationship between the oxygen mass flux and the effective diffusivity was experimentally determined using the tubes in each series of experiments, because a different relationship could occur due to a change in the settings of the measurement apparatus.

Figure 4 shows a flow diagram of the procedure used to determine the effective oxygen diffusivity in a GDL. First, the relationship between the apparent conductance of oxygen reaction area $C_{\mathrm{c}}$, and the oxygen mass flux $J_{\exp }$, is determined from experiments with the standard samples. This relationship is used as the boundary condition for the oxygen reaction area in the numerical calculation. Next, the initial effective diffusivity $D_{\text {eff }}$ in the GDL is set, and the oxygen flux $J_{\text {num }}$ and the oxygen concentration distribution is calculated. A two-dimensional oxygen concentration distribution is formed and there is some non-uniformity of the local oxygen mass flux at the oxygen absorption surface; therefore, $J_{\text {num }}$ is determined by integrating the local oxygen mass flux. Finally, the effective oxygen diffusivity is determined to within $\pm 0.5 \%$ by iterative calculation 
based on the difference between the numerical and measured values of the mass flux by varying the diffusivity for the GDL so that $J_{\text {num }}$ approaches $J_{\text {exp }}$.

\subsection{Experimental procedure for effective oxygen diffusivity measurement using galvanic cell oxygen absorber}

The comparison method was improved to measure the effective oxygen diffusivity of a single GDL containing moisture, and so particular care was taken to maintain the stability of the output characteristics of the galvanic cell. Specifically, variations in the output of the measurement apparatus under conditions without a sample were measured three times just before, during and after the measurement of the GDL test sample and the standard samples as shown in Fig. 5.

Effective oxygen diffusivity measurements were performed using the procedure listed below. The results are given in Figs. 5(a) and 5(b), which show the variation in GDL water saturation and output voltage during drying, respectively (steps (2) to (5), open circles). The closed circles enclosed by larger open circles in Fig. 5(b) represent the output of the oxygen absorber under steady-state electricity generation without a sample, the circles enclosed by open squares denote the output of the oxygen absorber with the test GDL, and the open diamonds represent the oxygen absorber output with standard samples.

(1) The oxygen absorber output under steady-state electricity generation was measured without the test material (measurement point $\mathrm{E}_{1}$ in Fig. 5(b)) prior to the GDL measurement.

(2) The test material filled with water was set in the holder and electricity generation was started.

(3) After a predetermined time interval, electricity generation was stopped and the weight of the test material was measured to obtain the average water saturation.

(4) The test material was immediately set back in the oxygen absorber and electricity generation was restarted.

(5) Steps (3)-(4) were repeated several times to obtain the relation between the water saturation and oxygen diffusion flux (measurement points between points $\mathrm{E}_{1}$ and $\mathrm{E}_{2}$ in Fig. 5(b)).

(6) Step (1) was carried out again (measurement point $\mathrm{E}_{2}$ in Fig. 5(b)).

(7) The experiment was performed with standard samples (measurement points located between points $E_{2}$ and $E_{3}$ in Fig. $5(\mathrm{~b}))$.

(8) Step (1) was carried out again (measurement point $\mathrm{E}_{3}$ in Fig. 5(b)).

Thus, the variations in the oxygen absorption characteristics of the measurement apparatus were measured and recorded by performing measurement procedures (1), (6) and (8) without the test material to determine the output stability of the galvanic cell during diffusivity measurement.

\subsection{Considerations for improvement of galvanic cell oxygen absorber output stability and corresponding validation methodologies}

Figure 6 shows a schematic diagram of the typical mass transfer resistances for diffusivity measurement. Three regions of mass transfer resistance were identified; $R_{\mathrm{Glv}}$ denotes the mass transfer resistance of the oxygen reaction area that is enclosed by the dot-dashed line, $R_{\mathrm{Gas}}$ is the oxygen diffusion resistance in the gas region between the test sample and oxygen reaction area with the oxygen limiting filter enclosed by the dashed line, and $R_{\mathrm{GDL}}$ is the oxygen diffusion resistance of the test sample. The overall resistance of the oxygen absorber is the sum of $\underline{R}_{\mathrm{Glv}}$ and $R_{\mathrm{Gas}}$. The typical ratio of the overall resistance of the oxygen absorber to $R_{\mathrm{GDL}}$ for a single dry GDL $\left(R_{\mathrm{Glv}}+R_{\mathrm{Gas}}\right) / R_{\mathrm{GDL}}$, is approximately 30 . The mass transfer resistance of a single dry GDL is low compared with the resistance of the oxygen absorber area, which indicates that the effect of a slight change in the mass transfer characteristics of the galvanic cell has a significant effect on the GDL diffusion coefficient measurement. As in our previous study [14], examination of the error factors did not effectively improve the output characteristics of the oxygen absorber, so that only the results with higher output stability obtained with low probability were used, corresponding to approximately $10 \%$ of the total number of measurements. Thus, structures that have effects on the change in the output characteristics of the galvanic cell oxygen absorber are the focus of this study to achieve high-precision measurements of the oxygen diffusivity. Specifically, the following items were identified as influential factors and were investigated with respect to changes in the galvanic cell configuration. 
2.4.1 Effect of electrolyte static pressure at oxygen reaction surface (degree of contact between carbon electrode film and electrolyte)

The carbon electrode film that contacts the electrolyte is very thin, so that oxygen permeation occurs easily. In addition, both contact with the electrolyte and prevention of electrolyte leaching is maintained by imparting hydrophobicity to the carbon electrode film. The pressure in the electrolyte container is adjusted to be equal to the surroundings. However, it is possible that the reaction area in the vicinity of the contact between the carbon electrode film and electrolyte changes after a relatively long time. That is, it is considered that the three phase area can change due to an increase in the static pressure exerted by the electrolyte on the carbon electrode film. The effect of changes in the degree of contact due to this pressure was examined by varying the electrolyte thickness, which is referred to as a liquid level difference.

\subsubsection{Effect of gap width between carbon electrode film and oxygen limiting filter and numerical analysis}

In our previous study [14], the oxygen limiting filter and the carbon electrode film were lapped over and situated close to each other. In such a situation, oxygen permeation from the oxygen limiting filter to the carbon electrode film would occur only locally. Therefore, the introduction of a gap between the two films would be expected to affect the oxygen diffusion characteristics. The effect of the gap width between the films on the oxygen diffusion characteristics and mass flux was examined and conditions to improve the stability were investigated experimentally and with numerical calculations

A three-dimensional numerical analysis using a Cartesian coordinate system was conducted with consideration of the actual configuration of the oxygen limiting filter with some small holes in a disk-shaped film. The oxygen flux at the oxygen reaction surface with oxygen concentration distribution and the oxygen concentration distribution were calculated for various gap widths. Figure 7 shows the calculation model with an oxygen limiting filter having nineteen 0.1 mm diameter holes through which oxygen permeates. The basic equation and boundary conditions are similar to those given in Section 2.2. The gap width between the carbon electrode film and the oxygen limiting filter was varied from 0.05 to $1.0 \mathrm{~mm}$ in the domain of $0.05 \leq z \leq 1.0$.

The local distribution of the oxygen mass fraction and the mass flux of oxygen at the oxygen reaction surface of the carbon electrode film were calculated for each gap width for the system shown in Fig. 7, given the apparent conductance of oxygen reaction area $C_{\mathrm{C}}$, which is selected as a typical characteristic of the galvanic cell oxygen absorber, and the diffusivity of a dry GDL, $D_{\text {eff. }}$ The relationship among the gap width, the local concentration distribution and the mass transfer rate for oxygen at the oxygen reaction surface of the carbon electrode film was determined.

\section{Results and discussion}

\subsection{Effect of static pressure of electrolyte at oxygen reaction surface (degree of contact between carbon electrode film} and electrolyte)

Figure 8 shows the output variation as a function of time in order to examine the effect of the static pressure exerted on the carbon electrode film of the galvanic cell oxygen absorber from the electrolyte for electrolyte thickness of 40 and $20 \mathrm{~mm}$. Experimental conditions other than the liquid thickness were not changed in order to maintain continuous measurement under constant conditions and take into account to minimize any gap-width effect. The output peaked at approximately $5000 \mathrm{~s}$ when the electrolyte thickness was $40 \mathrm{~mm}$ and then decreased gradually by $0.11 \mathrm{mV}$ (about 5.8\%) within approximately $5000 \mathrm{~s}$. When the electrolyte thickness was changed to $20 \mathrm{~mm}$, as shown between about 11000 and $21000 \mathrm{~s}$, the output variation was only $0.03 \mathrm{mV}$, which corresponds to $1.8 \%$. In addition, the output variation during approximately $8500 \mathrm{~s}$ was only $0.5 \%$ when the electrolyte thickness was $12 \mathrm{~mm}$. It is probable that the static pressure exerted by the electrolyte on the carbon electrode film caused a change in the reaction area and this influenced the output of the galvanic cell oxygen absorber. Since for an electrolyte thickness of $12 \mathrm{~mm}$, the change in the output was small, this was the value that was chosen for the diffusivity measurements. 


\subsection{Effect of gap width between carbon electrode film and oxygen limiting filter}

Figure 9 shows the oxygen concentration distribution obtained by numerical analysis for the gap width of $0.1 \mathrm{~mm}$. Figures 9(a) and 9(b) show the entire numerical calculation area and an enlarged view in the vicinity of the GDL and the oxygen limiting filter, respectively. A two-dimensional concentration distribution of oxygen was formed near the GDL, which is indicated by the dashed line in Fig. 9(b). Figure 9(b) also shows that a significant three-dimensional distribution of oxygen concentration formed, as shown in the circumferential areas of the light green colored cross section of small holes in the oxygen limiting filter.

Figure 10 shows the oxygen concentration distribution at the oxygen reaction surface, which is indicated by the red dashed line in Fig. 7. The gap width between the carbon electrode film and the oxygen limiting filter was changed from 0.05 to $1.0 \mathrm{~mm}$. The oxygen concentration near the small holes is locally high and the permeation area is not very uniform when the gap width is small. However, the distribution is almost smooth when the gap is wider. The average oxygen concentration distribution at the oxygen reaction surface has a gradual slope toward the center of the carbon electrode film. Thus, the oxygen concentration distribution becomes more uniform with increasing gap width.

Figure 11 shows the dependence of the oxygen transfer rate on the gap width. When the gap is narrow, the concentration distribution is not homogeneous and the diffusion resistance increases with decreasing gap width because only oxygen near the small holes is involved in the reaction. The oxygen transfer rate increases with gap width, and then becomes constant for a width of $0.5 \mathrm{~mm}$ or more. Since the oxygen transfer rate is particularly sensitive to small gap widths, and many factors could cause an unintentional change in the gap width during the measurements, a larger gap width of $1.0 \mathrm{~mm}$ was used in this study.

Figures 12(a) and 12(b) show the appearance of the carbon electrode film surface after the experiment, for gap widths of 0.1 and $1.0 \mathrm{~mm}$, respectively. The brighter circular regions in Fig. 12(a) indicate that the reaction occurred locally and close to small holes in the oxygen limiting filter. When the gap between the films was sufficiently large $(1.0 \mathrm{~mm})$, no such localized regions were observed, as shown in Fig. 12(b). This is consistent with the numerical calculation results. Therefore, it is considered that the output instability that occurred in the previous study [14] was because the gap between the carbon electrode film and the oxygen limiting filter was too small.

\subsection{Oxygen diffusivity measurement results and verification of measurement precision}

Figure 13 shows the oxygen diffusivity as a function of the average saturation $S$, using the improved settings for the liquid level difference and the gap width. The oxygen diffusivity determined in the previous study [14] is also shown for comparison. The average water saturation represents the fraction of pores occupied by liquid water in the porous medium; $100 \%$ saturation is when all of the pores are filled. The effective oxygen diffusivity increases with decreasing saturation from $100 \%$. In these measurements, the drying method involved a reduction in the degree of saturation by exposing the surface to ambient air and closing the galvanic cell-side surface. Therefore, the water content in the test material gradually decreases from the atmosphere-side surface. As a result, there is a difference between the average water saturation and that near the absorber; when evaporation proceeds and the average water saturation decreases to some extent, there are few effective paths for oxygen diffusion near the absorber due to the high local water saturation. Utaka et al. investigated the water distribution in a GDL with the same type of carbon paper using neutron radiography under similar drying conditions [16], and the results are consistent with those obtained in the present study. The effective diffusivity in the dry GDL $(S=0)$ is in the range $5.3 \times 10^{-6}$ to $7.4 \times 10^{-6} \mathrm{~m}^{2} / \mathrm{s}$, and that in the previous study [14] had a wider range of $2.5 \times 10^{-6}$ to $7.0 \times 10^{-6} \mathrm{~m}^{2} / \mathrm{s}$. Thus, an improved measurement precision was achieved in the present study.

The measurement precision for the effective diffusivity in a single GDL was investigated. In the previous report [14], the error factors were examined and the maximum value of the measurement error was estimated. The major error factors in common with this study are the effect of the density and temperature of the ambient air, and the effect of the specimen 
thickness. In addition, it was necessary to examine the temporal change in the oxygen absorber characteristics in the present study as a result of the adopted improvements.

The errors due to changes in the density and temperature of the ambient air, and the effect of the test sample thickness are expressed by Eqs. (11) and (12), respectively [14].

$$
\begin{aligned}
\left|\frac{\delta D_{1}}{D_{1}}\right| & =0.027 \\
\left|\frac{\delta D_{2}}{D_{2}}\right| & =0.027
\end{aligned}
$$

The stability of the galvanic cell oxygen absorber output was substantially improved by applying a suitable liquid level difference and gap width between the films, as discussed in Sections 3.1 and 3.2, respectively. Although only approximately $10 \%$ of the measurement results were used in the previous study [14], all the measurement data could be used in the present study.

Table 2 shows the maximum measured output fluctuations in the present study. The maximum difference in output between $E_{1}$ and $E_{2}$, which are shown in Fig. 5, is

$$
\left|\frac{E_{1}-E_{2}}{E}\right| \leq 0.0099
$$

The maximum error in the effective diffusivity due to temporal changes in the oxygen absorber characteristics is

$$
\left|\frac{\delta D_{3}}{D_{3}}\right| \leq 0.213
$$

Therefore, temporal changes in the oxygen absorber characteristics have the most significant effect on the measurement precision. The total error in the effective diffusivity under dry conditions is

$$
\text { error }=\sqrt{\left|\frac{\delta D_{1}}{D_{1}}\right|^{2}+\left|\frac{\delta D_{2}}{D_{2}}\right|^{2}+\left|\frac{\delta D_{3}}{D_{3}}\right|^{2}}=0.22 .
$$

Figure 13 shows a total of 17 diffusivity measurement results that are within $\pm 18 \%$, which confirms that they satisfy Eq. (18) and are sufficiently accurate. Moreover, the effective oxygen diffusivity in the dry GDL was in approximate agreement with that measured experimentally using a multilayered GDL, as reported by Utaka et al. $\left(6.5 \times 10^{-6} \mathrm{~m}^{2} / \mathrm{s}\right)[13]$ and Zamel et al. $\left(5.3 \times 10^{-6} \mathrm{~m}^{2} / \mathrm{s}\right)$ [11], although the specifications of these GDLs were different.

The GDL specimen containing moisture has a larger diffusion resistance than a dry GDL, so that the measurement accuracy is high for a GDL containing moisture. For example, the measurement error is calculated to be $5.9 \%$ when the average water saturation is $S=24 \%$. However, Fig. 13 shows that the measurement results under wet conditions have comparatively large variations. This may be due to the irregular formation of oxygen diffusion paths for each sample because the microscopic structure of the GDL is not uniform. Therefore, the differences among samples would be significant because even a small amount of moisture can easily cause liquid water to obstruct the permeation of oxygen. This is because the carbon fibers in the GDL are located almost in the direction perpendicular to oxygen permeation and the liquid water distribution is non-uniform.

\section{Conclusions}

To improve the measurement precision for the oxygen diffusion characteristics of a PEFC GDL with/without the presence of moisture, factors that should be improved in order to increase the output stability of the galvanic cell oxygen absorber were examined. Two factors were focused on: the degree of contact between the carbon electrode film and the electrolyte, and the gap width between the carbon electrode film and the oxygen limiting filter. The accuracy of the diffusivity measurement was 
improved by changing the configuration of the galvanic cell oxygen absorber measurement apparatus. The results are summarized as follows.

(1) The degree of contact between the carbon electrode film and the electrolyte is expected to affect the areal distribution of reactions, and as such, influence the stability of the galvanic cell output characteristics. To investigate this, the effect of the electrolyte thickness was investigated, since this determines the amount of static pressure exerted on the carbon electrode. An electrolyte thickness less than $12 \mathrm{~mm}$ was found to lead to stable output.

(2) The effect of the gap width between the carbon electrode film and the oxygen limiting filter was examined. Small gap widths were found to have a significant effect on the output characteristics, so a comparatively large gap width of $1 \mathrm{~mm}$ was used to improve the stability in the present study.

(3) A comparison was made between the results of effective oxygen diffusivity measurements performed using the improved galvanic cell oxygen absorber configuration and previous results [14]. It was found that using the improved setup led to a decrease in the error from 50 to $20 \%$ for a dry GDL, even though such a GDL has a very low diffusion resistance and is therefore subject to large errors.

\section{Acknowledgment}

This work was supported in part by a Grant-in-Aid for Scientific Research [(B)23360097] from the Japan Society for the Promotion of Science (JSPS) and the Strategic International Collaborative Research Program of the Japan Science and Technology Agency (JST).

\section{References}

[1] Henry, J. P., Chennakesavan, B. and Smith, J. M., "Diffusion Rates in Porous Catalysts", AIChE J., Vol. 7, (1961), pp.10-12.

[2] Masamune, S. and Smith, J. M., "Pore Diffusion in Silver Catalysts", AIChE J., Vol. 8 (1962), pp. 218-221.

[3] Wakao, N. and Funaki, T., "Effective Diffusivity in Catalyst Pellets", Chemical Engineering (Japan), Vol. 31, No. 5 (1967), pp. 485-490.

[4] Hamai, S. and Mitani, M., "Research on Gaseous Diffusion Phenomena in Various Porous Catalysts (Measurement of Effective Diffusion Coefficient by Frontal Chromatography)", Chemical Engineering (Japan), Vol. 34, No. 10 (1970), pp. 1086-1092.

[5] Gibilaro, L. G. and Waldram, S. P., "Measurement of Effective Binary Gas Diffusion Coefficients in Porous Catalysts", J. Catal., Vol. 67, Issue 2 (1981), pp. 392-401.

[6] Mezedur, M. M., Kaviany, M. and Moore, W., "Effect of Pore Structure, Randomness and Size on Effective Mass Diffusivity”, AIChE J., Vol. 48, No. 1 (2002), pp. 15-24.

[7] Kramer, D., Freunberger, S. A., Flückiger, R., Schneider, I. A., Wokaun, A., Büchi, F. N. and Scherer, G. G., "Electrochemical diffusimetry of fuel cell gas diffusion layers", J. Electroanal. Chem., Vol. 612, Issue 1, (2008), pp. 63-77.

[8] Flückiger, R., Freunberger, S. A., Kramer, D., Wokaun, A., Scherer, G. G. and Büchi, F. N., "Anisotropic, effective diffusivity of porous gas diffusion layer materials for PEFC", Electrochim. Acta, Vol. 54, Issue 2, (2008), pp. 551-559.

[9] Baker, D. R., Wieser, C., Neyerlin, K. C. and Murphy, M. W., "The Use of Limiting Current to Determine Transport Resistance in PEM Fuel Cells", ECS Trans., Vol. 3, Issue 1, (2006), pp. 989-999.

[10] Hwang, G. S. and Weber, A. Z., "Effective-Diffusivity Measurement of Partially-Saturated Fuel-Cell Gas-Diffusion Layers", J. Electrochem. Soc., Vol. 159, Issue 11, (2012), pp. F683-F692.

[11] Zamel, N., Astrath, N. G. C., Li, X., Shen, J., Zhou, J., Astrath, F. B. G., Wang, H. and Liu, Z., "Experimental measurements of effective diffusion coefficient of oxygen- nitrogen mixture in PEM fuel cell diffusion media", Chem. Eng. Sci., Vol. 65, Issue 2 (2010), pp. 931-937. 
[12] Chan, C., Zamel, N., Li, X. and Shen, J., "Experimental measurement of effective diffusion coefficient of gas diffusion layer/microporous layer in PEM fuel cells", Electrochim. Acta, Vol. 65, Issue 30 (2012), pp. 13-21.

[13] Utaka, Y., Tasaki, Y., Wang, S., Ishiji, T. and Uchikoshi, S., "Method of measuring oxygen diffusivity in microporous media", Int. J. Heat Mass Transfer, Vol. 52, Issue 15-16, (2009), pp. 3685-3692.

[14] Utaka, Y., Iwasaki, D., Tasaki, Y. and Wang, S., "Measurement of effective oxygen diffusivity in microporous media containing moisture", Heat Transfer Asian Res., Vol. 39, Issue 4 (2010), pp. 262-276.

[15] Utaka, Y., Hirose, I. and Tasaki, Y., "Characteristics of oxygen diffusivity and water distribution by X-ray radiography in microporous media in alternate porous layers of different wettability for moisture control in gas diffusion layer of PEFC”, Int. J. Hydrogen Energy, Vol. 36, Issue 15 (2011), pp.9128-9138.

[16] Utaka, Y., Tasaki, Y., Wang, S., Iwasaki, D., Waki, N., Kubo, N., Shinohara, K., Boillat, P., Frei, G., Oberholzer, P., Scherer, G. G. and Lehmann, E. H., "Mass Transfer Characteristics in Porous Media Applying Simultaneous Measurement Method of Water Visualization by Neutron radiography and Oxygen Diffusivity", Trans. Jpn. Soc. Mech Eng, Series B, Vol. 76, No. 771 (2010), pp. 1964-1972. 


\section{Figures and tables}

Figure 1 Experimental apparatus with galvanic cell oxygen absorber.

Figure 2 Complete experimental setup.

Figure 3 Calculation model for oxygen absorber.

Figure 4 Flow diagram for numerical calculation.

Figure 5 Variation of water saturation level in GDL and output voltage.

(a) Water saturation (b) Output voltage ( $\bigcirc$ : Output voltage without sample, $\square$ : Oxygen diffusivity measurement in GDL, $\diamond:$ Oxygen diffusivity measurement using circular pipe)

Figure 6 Schematic diagram of mass transfer resistance.

Figure 7 Calculation model for oxygen absorber.

Figure 8 Effect of static pressure on output voltage.

Figure 9 Distribution of oxygen concentration determined by numerical calculations.

(a) Full view.

(b) Enlarged view.

Figure 10 Mass fraction of oxygen at carbon electrode film surface.

Figure 11 Relationship between oxygen transfer rate and gap width between carbon cathode film and oxygen limiting filter.

Figure 12 Appearance of carbon electrode film after experiment.

(a) Gap of $0.1 \mathrm{~mm}$ between films.

(b) Gap of $1.0 \mathrm{~mm}$ between films.

Figure 13 Variation of effective oxygen diffusivity with average saturation.

Table 1 Specifications of GDL porous media test sample.

Table 2 Variation of output voltage. 
Table 1 Specifications of GDL porous medium test sample.

\begin{tabular}{|c|c|c|c|c|}
\hline Test sample & Porosity & Representative Pore Diameter $[\mu \mathrm{m}]$ & Permeability $\left[\mu \mathrm{m}^{2}\right]$ & Thickness $[\mu \mathrm{m}]$ \\
\hline Carbon Paper GDL & 0.78 & 32 & 3.2 & 370 \\
\hline
\end{tabular}

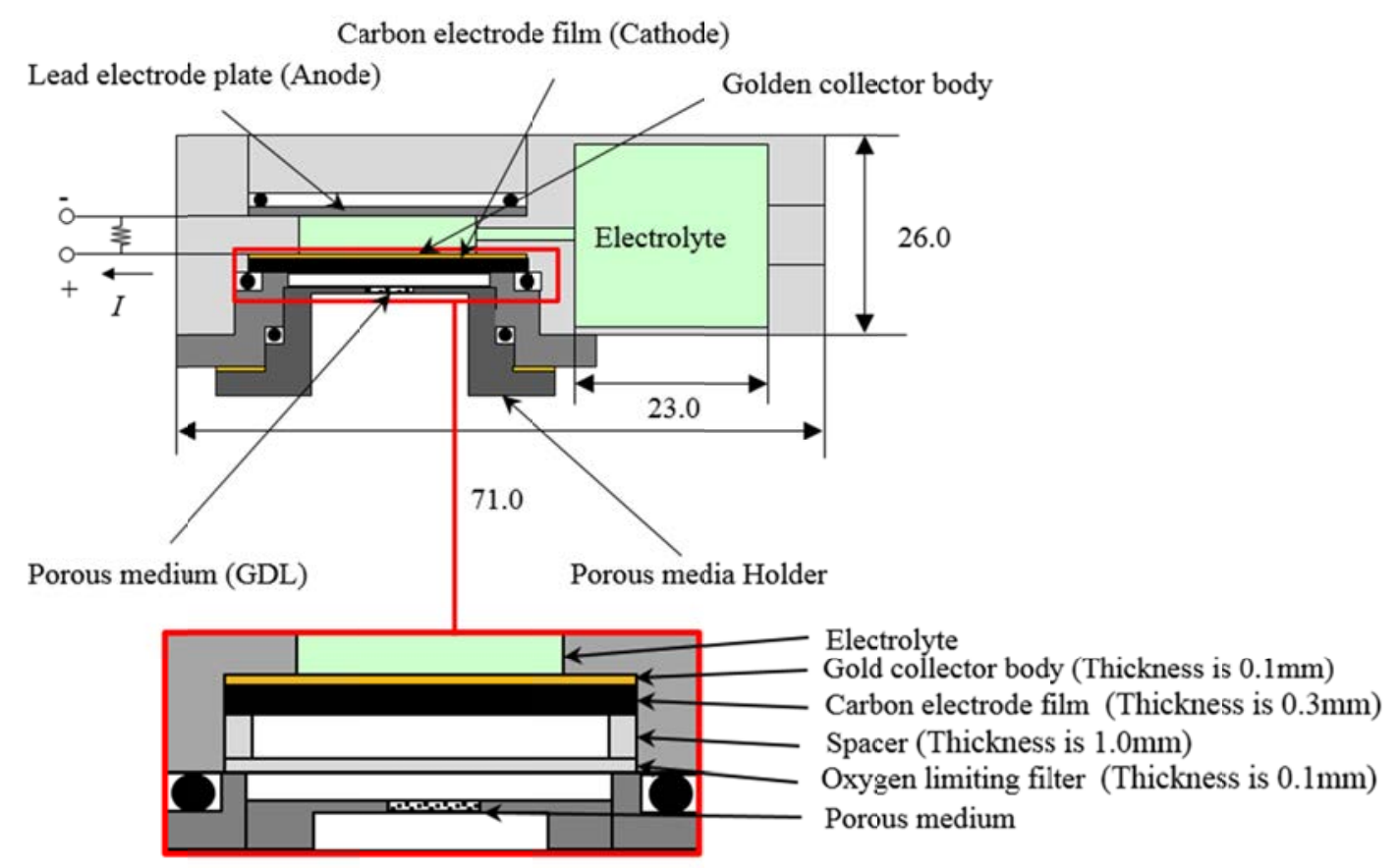

Figure 1 Experimental apparatus with galvanic cell oxygen absorber.

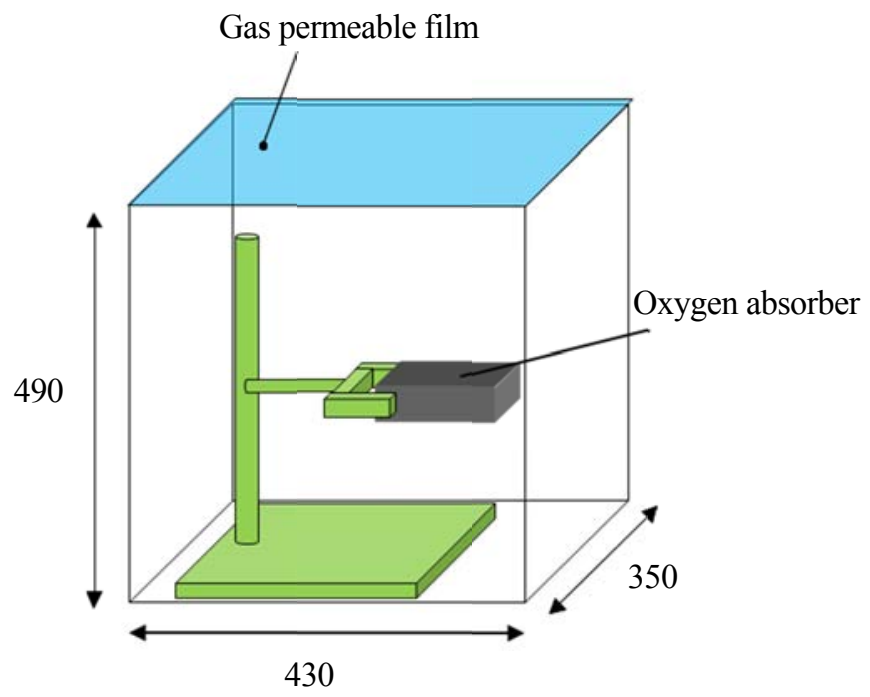

Figure 2 Complete experimental setup. 


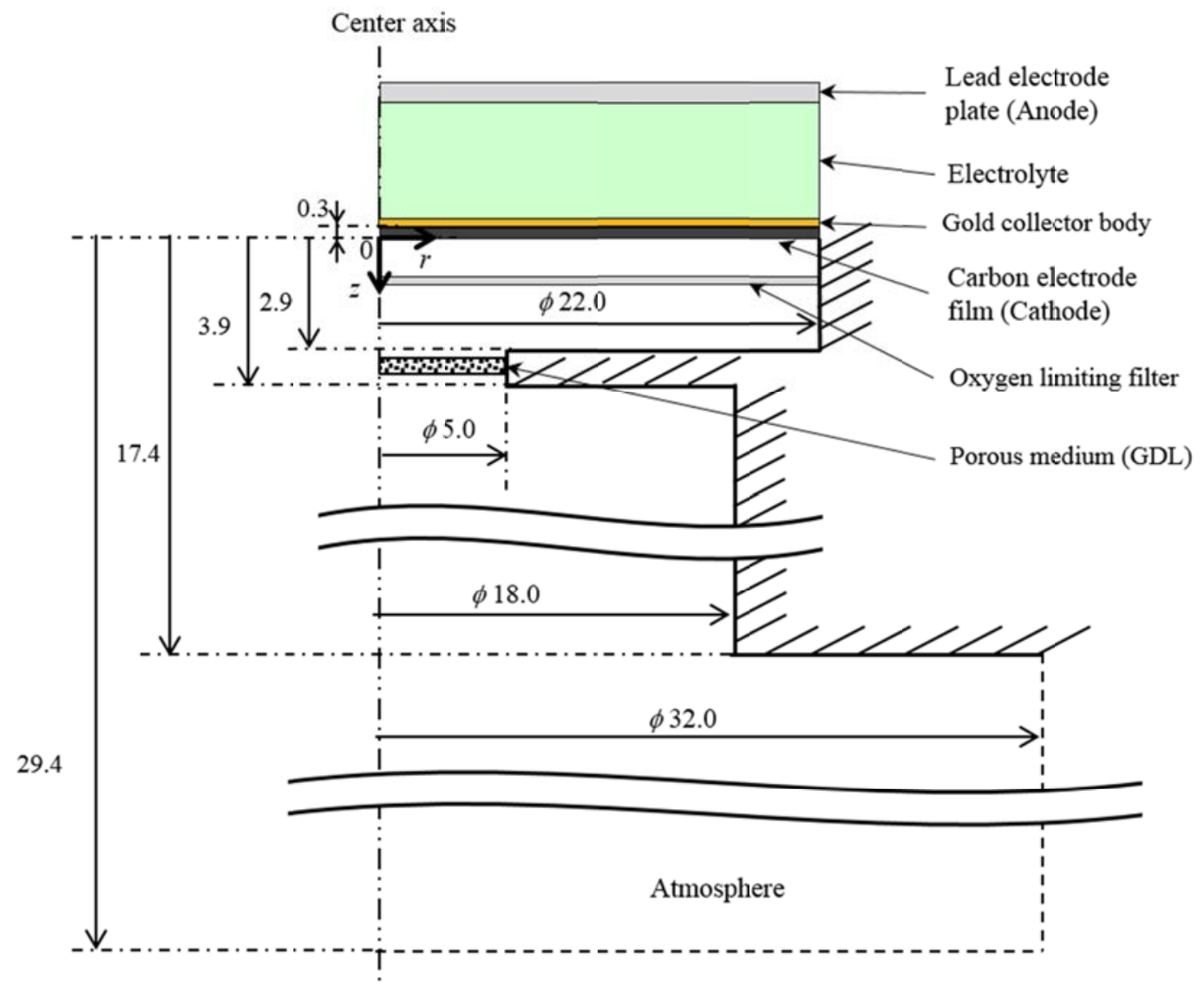

Figure 3 Calculation model for oxygen absorber.

Calculate the oxygen diffusivity $D_{\text {es }}$

Determine the oxygen diffusion conductance $C_{\mathrm{C}}$ in the carbon electrode film

by $C_{c}=f\left(J_{\text {esp }}\right)$

Give the initial oxygen diffusivity $D_{\text {ett }}$ in the specimen

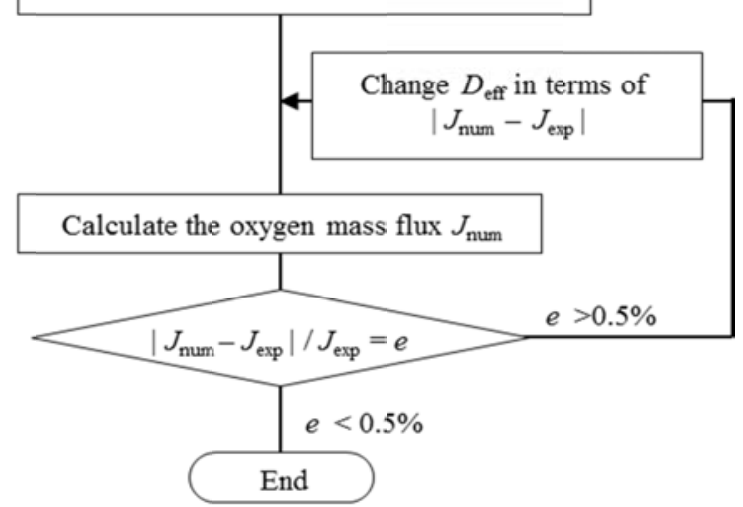

Figure 4 Flow diagram for numerical calculation. 


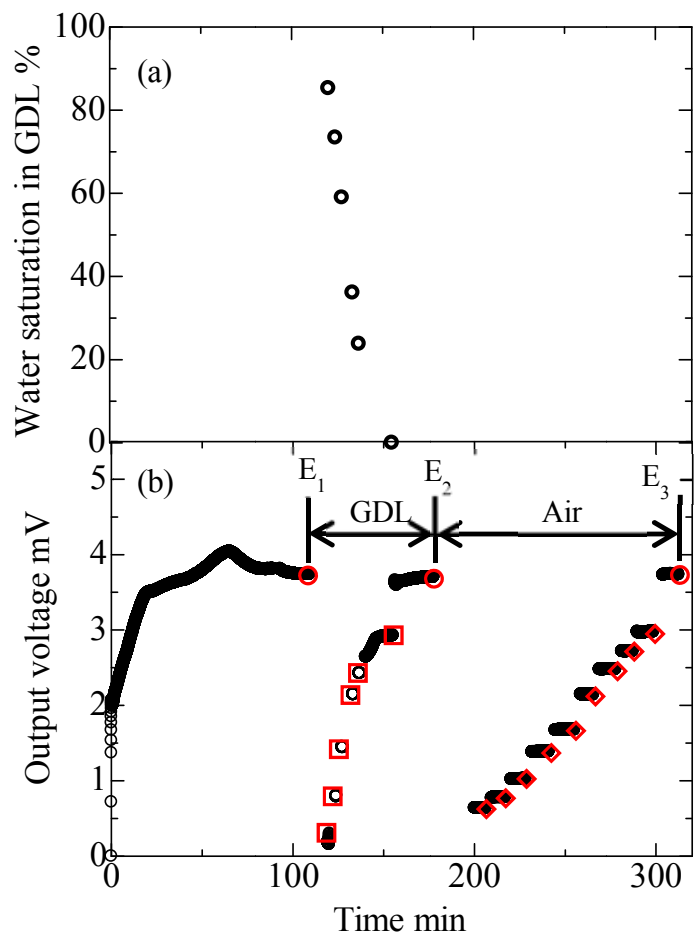

Figure 5 Variation of water saturation in GDL and output voltage (a) Water saturation (b) Output voltage ( $\bigcirc$ : Output oltage without sample, $\square$ : Oxygen diffusivity measurement in GDL, $\diamond$ : Oxygen diffusivity measurement using

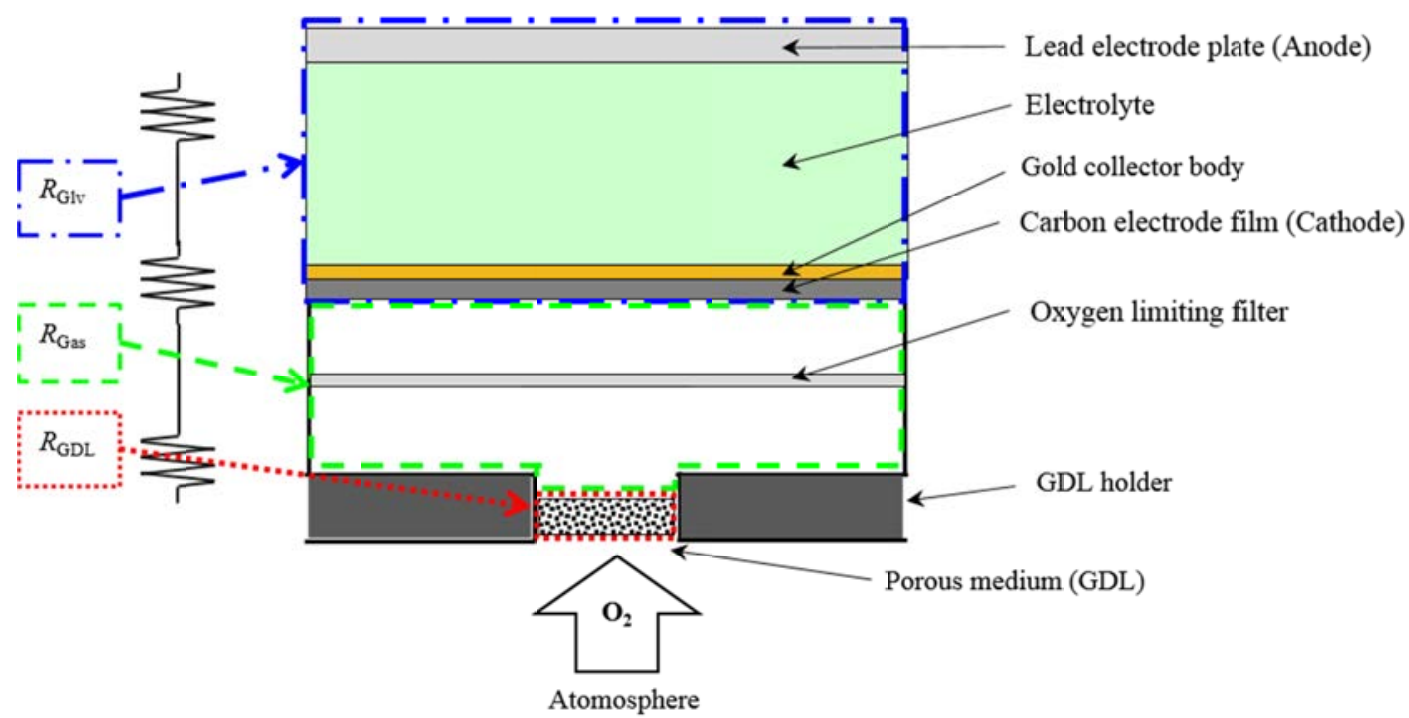

Figure 6 Schematic diagram of mass transfer resistance. 


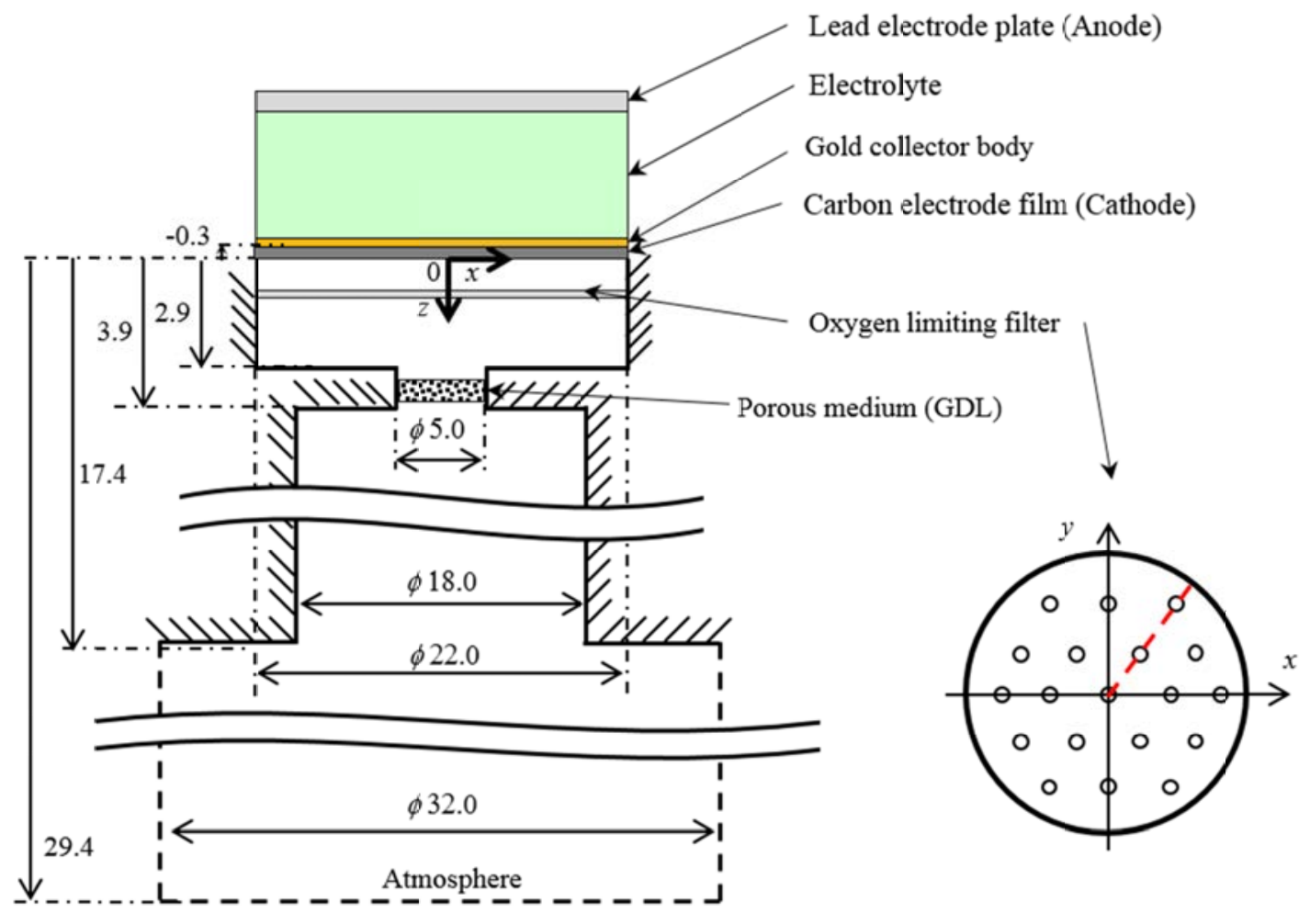

Figure 7 Calculation model for oxygen absorber.

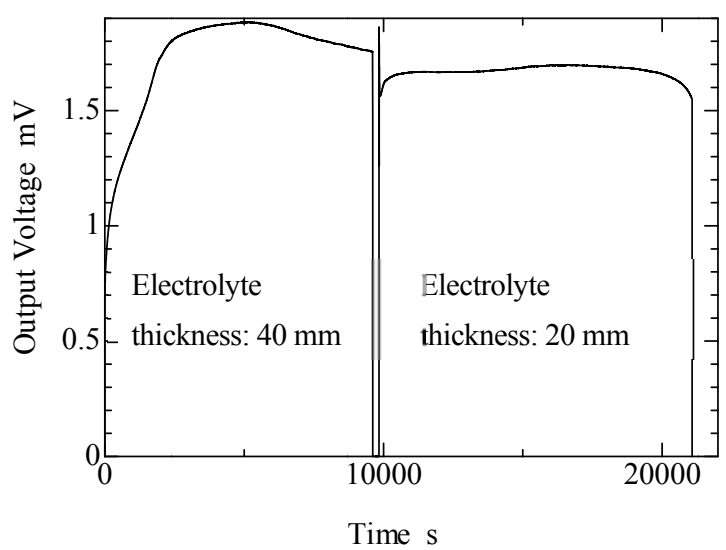

Figure 8 Effect of static pressure on output voltage. 


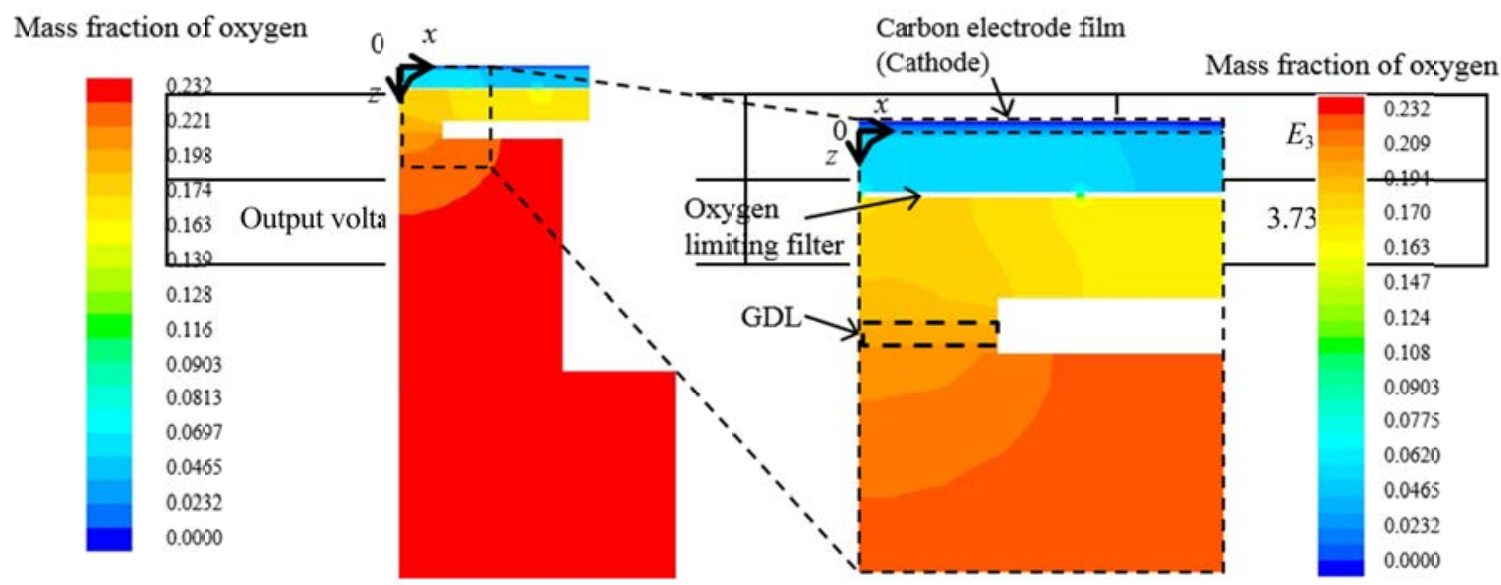

(a) Entire oxygen concentration distribution.

(b) Enlarged view of oxygen concentration distribution.

Figure 9. Distribution of oxygen concentration obtained by numerical calculations.

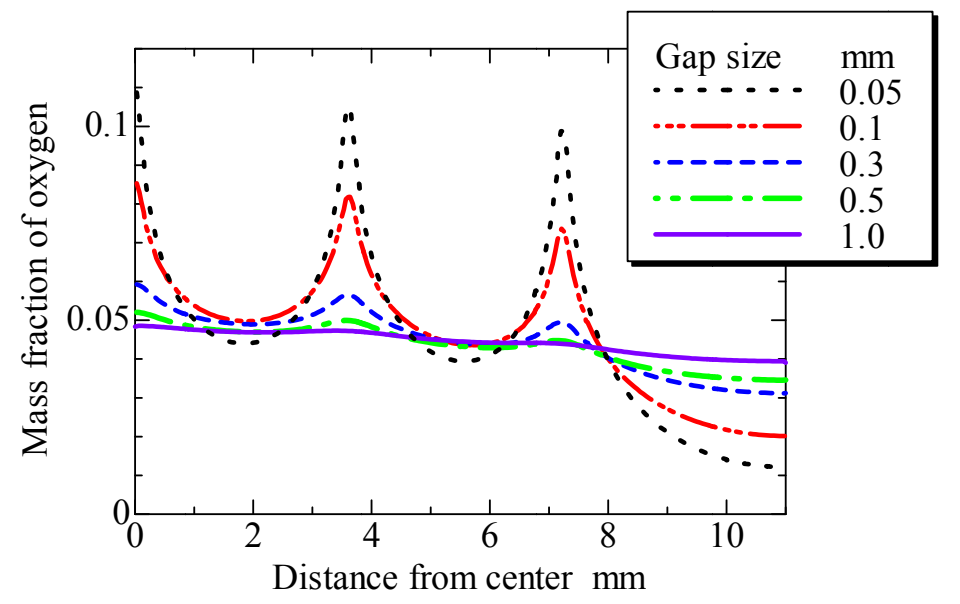

Figure 10 Mass fraction of oxygen at carbon electrode film surface. 


\begin{tabular}{|c|c|c|c|}
\hline Change in output \% & $\begin{array}{c}- \\
\text { Table 2 Variation of output voltage }\end{array}$ & $\frac{E_{1}-E_{2}}{E_{1}} \times 100=-0.99$ \\
\hline
\end{tabular}

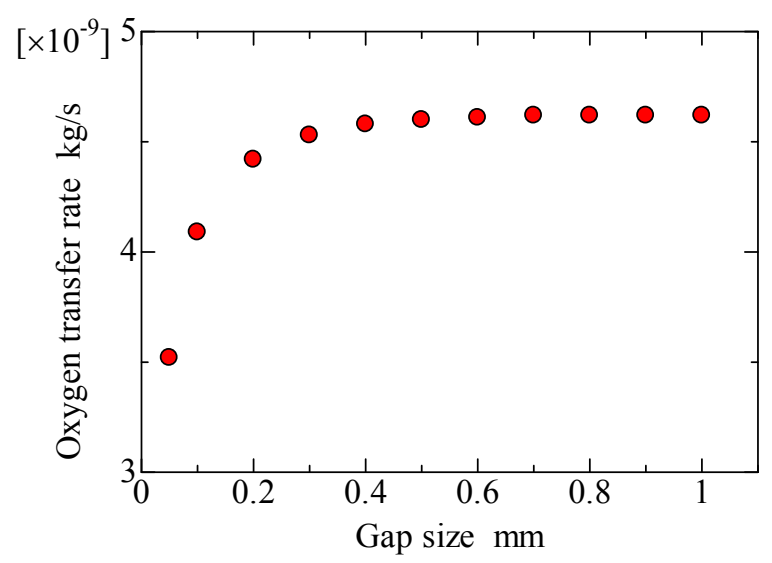

Figure 11 Relationship between oxygen transfer rate and gap width between carbon cathode film and oxygen limiting filter.

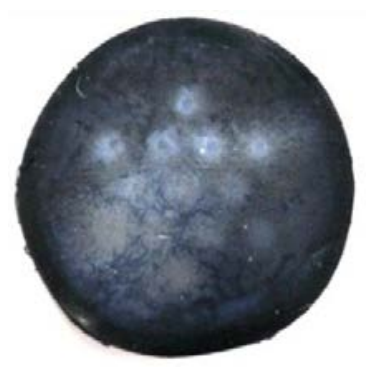

(a) Gap width of $0.1 \mathrm{~mm}$ between films.

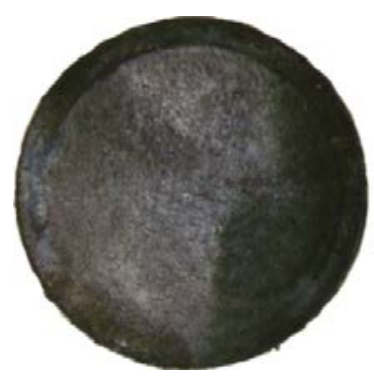

(b) Gap width of $1.0 \mathrm{~mm}$ between films.

Figure 12 Appearance of carbon electrode film after the experiment. 


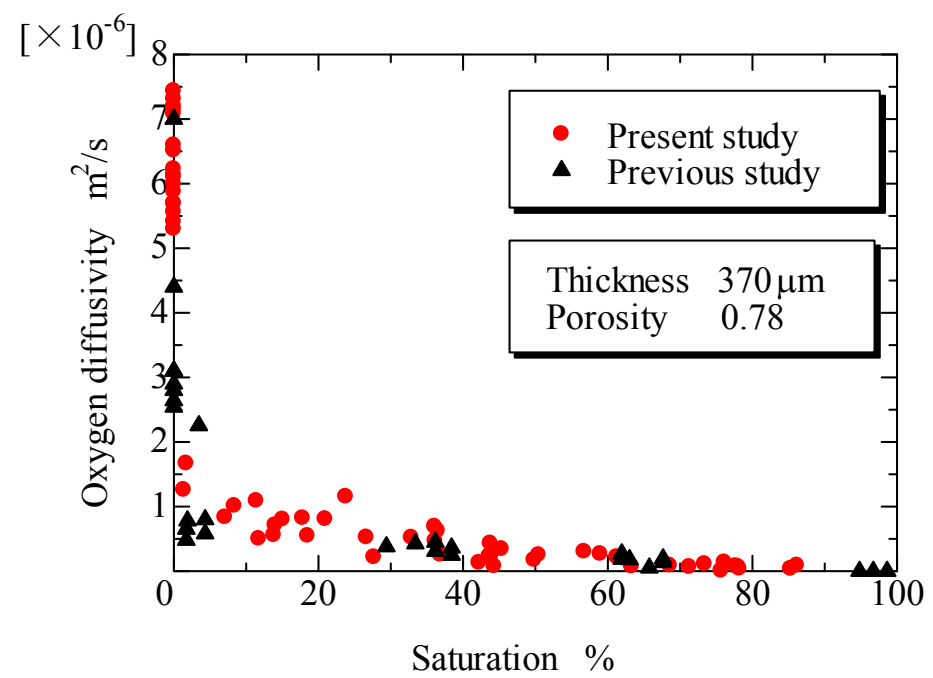

Figure 13 Variation of effective oxygen diffusivity with average saturation. 